The effectiveness of mifamurtide in osteogenic sarcoma treatment cannot be considered as assessed due to the small sample size.

No conflict of interest.

\section{DGI-035 EVALUATION OF THE SYSTEMIC TOXICITY OF DOXORUBICIN AFTER HEPATIC IODIZED OIL CHEMOEMBOLIZATION IN HEPATOCELLULAR CARCINOMA PATIENTS}

\author{
doi:10.1136/ejhpharm-2013-000276.301
}

${ }^{\prime} \mathrm{C}$ Fouche, 'MT Baylatry, ${ }^{2} \mathrm{~J}$ Cazejust, 'AC Joly. 'Saint-Antoine Hospital (APHP), Pharmacy, Paris, France; ' 2 Saint-Antoine Hospital (APHP), Interventional Radiology, Paris, France

Background Chemoembolization of iodized oil into a hepatic tumour (hioCE) is a locoregional medical technique that consists of delivering selectively into tumour-feeding arteries, an anticancer drug emulsified in iodized oil followed by an occlusive agent (embolization agent). It enables higher intra-tumour drug concentrations to be obtained compared to intravenous treatment, with blood vessel occlusion causing local necrosis. hioCE using doxorubicin at $50 \mathrm{mg} / \mathrm{m}^{2}$ is effective in the palliative treatment of hepatocellular carcinoma (HCC) with significant survival benefit compared with best supportive care. To our knowledge, no study has evaluated systemic doxorubicin toxicity after hioCE.

Purpose To evaluate systemic doxorubicin toxicity in HCC patients treated by hioCE.

Materials and Methods A 3-year retrospective study was performed in the Radiology and Pharmacy departments. Toxicity was assessed using WHO criteria. Data were collected from Chimio software and patient medical records. Mann Whitney and Chi2 tests were used.

Results 94 HCC patients were treated with hioCE using doxorubicin. Median age was 64 years [28-89]. Toxicity occurred in 69 patients (73\%). Main toxicities were digestive disorders (34 patients; 16 grade $3-4)$, cardiotoxicity (16 patients; 10 grade $3-4$ ) and alopecia (13 patients; 8 grade 3-4). No statistical relationship was found between patient characteristics (age, sex, body mass index, medical and surgical history), HCC aetiology or characteristics, Child-Pugh score or hioCE practise and the occurrence or gravity of doxorubicin toxicity.

Conclusions More than half of the patients suffered doxorubicin toxicity after hioCE suggesting doxorubicin passed into the systemic circulation. Studies showed that the doxorubicin-iodized oil mixture was unstable. Although hioCE with doxorubicin is effective in HCC and doxorubicin toxicity occurring in our patients was less severe than that of intravenous doxorubicin administration, doxorubicin tolerance after hioCE is debatable. The use of an anticancer drug that was more stable with iodized oil could decrease the passage of the drug into the systemic circulation. The use of doxorubicin-eluting beads for chemoembolization is much more expensive but could also be an alternative.

No conflict of interest.

\section{DGI-036 EVOLUTION OF ANTIFUNGAL CONSUMPTION IN A GENERAL HOSPITAL}

doi:10.1136/ejhpharm-2013-000276.302

'E Rodríguez Molíns, 'M Alcalá Galán, ${ }^{2} \mathrm{~A}$ Tenorio-Abreu, 'E Sánchez Gómez, 'I Pérez Díaz, 'Y Arco Prados. 'Juan Ramon Jimenez Hospital., Pharmacy Department, Huelva, Spain; 'Juan Ramon Jimenez Hospital., Microbiology Department, Huelva, Spain

Background Antifungal treatment is an important part of global expenditure. A significant increase in the use of these drugs does entail a higher cost.

It is hoped that the use of these drugs will continue to increase each year. It is important to know the drug use distribution through the different units and the monetary cost in order to put forward pharmacist interventions.

Purpose To describe the evolution of expenditure on, and consumption of, caspofungin, voriconazole, amphotericin B and fluconazole and significant fungaemia from 2009 to 2011.

Materials and Methods Observational, retrospective study, carried out in a General Hospital.

The consumption for every single patient of caspofungin, voriconazole, liposomal amphotericin B and fluconazole, from 2009 to 2011, were obtained from the Pharmacy Department Software databases (Langtools). Average prices were used to calculate the financial impact. In the microbiology department, blood cultures were done for every patient treated with these drugs for fungal isolates.

Results Pharmaceutical spending on these four drugs versus general expenditure was $1.53 \%, 1.04 \%$ and $1.00 \%$ for the years 2009 , 2010 and 2011 respectively. The evolution of consumption in units (including all presentations) and expenditure is shown in the following table (table 1)

The total consumption of the main services in the study period is shown in the following table (table 2).

The number of yeasts isolated from blood cultures was 20,19 and 21 for the years 2009, 2010 and 2011 respectively, representing $2.48 \%$ of all positive blood cultures.

Abstract DGI-036 Table 1

\begin{tabular}{|c|c|c|c|c|c|c|}
\hline & Units 2009 & Spending $2009(€)$ & Units 2010 & Spending $2010(€)$ & Units 2011 & Spending 2011(€) \\
\hline Caspofungin 50 mg vial & 426 & $198,935.95$ & 218 & $94,934.03$ & 148 & $64.714,53$ \\
\hline Voriconazole 200 mg vial & 541 & $41,914.25$ & 468 & $37,146.39$ & 731 & $44.453,75$ \\
\hline Liposomal Amphotericin B 50 mg vial & 1456 & $142,091.04$ & 1353 & $132,042.78$ & 1792 & $174.885,93$ \\
\hline Fluconazole $400 \mathrm{mg}$ vial & 2759 & $4,566.79$ & 2701 & $4,799.73$ & 2623 & $4.711,38$ \\
\hline Total & 5182 & $387,508.03$ & 4740 & $268,922.93$ & 5294 & $288,765.59$ \\
\hline Total pharmaceutical expenditure & & $25,310,713$ & & $25,824,331$ & & $28,771,067$ \\
\hline
\end{tabular}

Abstract DGI-036 Table 2

\begin{tabular}{|c|c|c|c|c|c|c|}
\hline & Units 2009 & Spending $2009(€)$ & Units 2010 & Spending $2010(€)$ & Units 2011 & Spending 2011(€) \\
\hline Caspofungin 50 mg vial & 426 & $198,935.95$ & 218 & $94,934.03$ & 148 & $64,714.53$ \\
\hline Voriconazole $200 \mathrm{mg}$ vial & 541 & $41,914.25$ & 468 & $37,146.39$ & 731 & $44,453, .75$ \\
\hline Liposomal Amphotericin B 50 mg vial & 1456 & $142,091.04$ & 1353 & $132,042.78$ & 1792 & $174,885.93$ \\
\hline Fluconazole $400 \mathrm{mg}$ vial & 2759 & $4,566.79$ & 2701 & $4,799.73$ & 2623 & $4,711.38$ \\
\hline Total & 5182 & $387,508.03$ & 4740 & $268,922.93$ & 5294 & $288,765.59$ \\
\hline Total pharmaceutical expenditure & & $25,310,713$ & & $25,824,331$ & & $28,771,067$ \\
\hline
\end{tabular}

